Cahiers $d u$ MONDE RUSSE

\section{Cahiers du monde russe}

Russie - Empire russe - Union soviétique et États indépendants

$52 / 4 \mid 2011$

Varia

\title{
Olaf Mertelsmann, ed., Central and Eastern European Media Under Dictatorial Rule and in the Early Cold War
}

Larissa Zakharova

\section{(2) OpenEdition}

12 Journals

Édition électronique

URL : http://journals.openedition.org/monderusse/7640

DOI : $10.4000 /$ monderusse. 7640

ISSN : $1777-5388$

Éditeur

Éditions de l'EHESS

Édition imprimée

Date de publication : 20 décembre 2011

Pagination : 787-790

ISBN : 978-2-7132-2353-2

ISSN : $1252-6576$

Référence électronique

Larissa Zakharova, «Olaf Mertelsmann, ed., Central and Eastern European Media Under Dictatorial Rule and in the Early Cold War », Cahiers du monde russe [En ligne], 52/4 | 2011, mis en ligne le 03 décembre 2012, Consulté le 23 septembre 2020. URL : http://journals.openedition.org/monderusse/ 7640 ; DOI : https://doi.org/10.4000/monderusse.7640

Ce document a été généré automatiquement le 23 septembre 2020.

(c) École des hautes études en sciences sociales 


\title{
Olaf Mertelsmann, ed., Central and Eastern European Media Under Dictatorial Rule and in the Early Cold War
}

\author{
Larissa Zakharova
}

\section{RÉFÉRENCE}

Olaf MERTELSMANn, ed., Central and Eastern European Media Under Dictatorial Rule and in the Early Cold War. Francfort-sur-le-Main : Peter Lang (Tartu Historical Studies, 1), 2011, 234 p.

Une ancienne plaisanterie soviétique dit que la seule vraie information contenue dans la Pravda est la date à la une. Les auteurs de cet ouvrage cherchent à montrer l'imposition du modèle soviétique de la diffusion de l'information dans les démocraties populaires, tout en se plaçant dans une perspective comparatiste avec l'Italie fasciste, l'Allemagne nazie et l'Autriche. Les médias sont présentés comme les instruments aussi bien que les reflets de la soviétisation et de la Guerre froide en Europe centrale et orientale. Comme c'est souvent le cas lors de la publication d'actes de colloques, les quatorze contributions de cet ouvrage issues d'une rencontre organisée à l'université de Tartu en 2005, sont de qualité très inégale. Le livre lui-même n'est pas organisé thématiquement. Deux articles sont consacrés au cinéma, deux à la télévision, un à la radio, un autre étudie ensemble cinéma et radio, les autres contributions traitant de la presse.

2 La mise en place de nouvelles procédures de censure constitue un thème transversal du livre. Le directeur de l'ouvrage, Olaf Mertelsmann, souligne la multitude des modèles de la censure dont la sévérité varie d'un pays à l'autre. Examiner ce thème pour l'ensemble du bloc aurait, d'après lui, gommé les nuances et effacé les différences. Cependant, en 
lisant les contributions, on a l'impression que les différences qui sont ici mises au jour résultent surtout de la variété des méthodes d'enquête des auteurs. Pour certains d'entre eux, la soviétisation des médias est la preuve de l'imposition du régime totalitaire, quand aucune marge de manœuvre n'est envisageable. Ainsi, Marietta Stankova, Peter Gross et Dorina Orzac traitant de la Bulgarie et de la Roumanie à la fin des années 1940 - début des années 1950 racontent comment la liberté fut étouffée dans la presse à la suite de la promulgation de décrets et de la création d'institutions en charge de la censure. Leur approche par le haut ne laisse pas de place pour une réflexion sur la réception de la nouvelle presse, sur l'intériorisation de la nouvelle grille d'analyse et des clichés par les lecteurs, ou sur la possibilité de lire entre les lignes. Ces auteurs, ainsi que Sirje Olesk et Andrej Školkay qui examinent l'adaptation des intellectuels à la nouvelle presse littéraire en Estonie et en Slovaquie, ne pensent pas en termes de techniques de pouvoir, de jeux avec les contraintes, ils ignorent le flou autour des normes et des prescriptions.

3 Les contributions de Jürgen Wilke, Jane Leftwich Curry et de Judith Devlin, proposent une approche plus fine du fonctionnement de la censure. Jürgen Wilke examine les instructions données à la presse comme constituant un dispositif de contrôle des médias en RDA. En remontant à la Première Guerre mondiale où ces instructions apparaissent pour la première fois, il montre que les différentes façons de transmettre ces instructions avec des sujets à couvrir et à éviter (transmission orale avec prise de notes, transmission par téléphone, par télégraphe ou instructions rédigées et diffusées sous forme de brochures imprimées) sont évocatrices des changements de régimes politiques. Ainsi, la RDA, qui pousse la précision des instructions à l'extrême, dépasse en dureté la censure de l'Allemagne nazie. Jane Leftwich Curry met en évidence les problèmes et les contradictions de la censure en Pologne dans les années 1940 et 1950. Les autorités se trouvent devant un dilemme : il leur faut recruter des censeurs parmi les ouvriers et paysans ; ils craignent que ceux-ci ne soient pas capables de lire entre les lignes. Vu le flou dans la définition du négatif et de l'interdit, la censure porte essentiellement sur les phrases équivoques et ambiguës, les sous-entendus, les lectures et les interprétations possibles. Elle a des implications financières pour les journalistes qui sont payés au nombre d'articles publiés et qui ont donc un intérêt pécuniaire à pratiquer l'autocensure. Enfin, Judith Devlin analyse la façon dont l'image de Stalin est construite dans le cinéma soviétique en présentant la censure comme un processus interactif: les instructions viennent certes d'en haut, mais elles doivent être interprétées, interprétations qui différent non seulement entre les censeurs, mais aussi parmi les scénaristes et les réalisateurs, d'où une marge d'erreur et les répressions qui s'ensuivent, modelant à leur tour l'image à projeter. Les idées concurrentes et divergentes qui s'expriment quant au résultat attendu d'un film, l'autocensure et les stratégies de communication dans le dos des censeurs montrent à quel point la censure cinématographique était un phénomène complexe et ambigu.

Deux contributions cherchent à dépasser l'image de l'audience passive des médias en montrant comment les individus choisissent leurs sources d'information. Roland Graf s'intéresse au cas paradoxal de la lecture de la presse communiste en Autriche de 1945 à 1959: le nombre de lecteurs des journaux communistes dépasse de cinq fois le nombre de ceux qui votent pour le PC. Le succès journalistique, qui témoigne d'un échec de la propagande, s'explique par la tendance à couvrir de façon presque apolitique des sujets qui préoccupent «les gens ordinaires». Olaf Mertelsmann examine, de son côté, les sources alternatives d'information en Estonie dans 
l'après-guerre: les émissions radiophoniques occidentales et les films dits «de trophée ». Ces derniers permettent d'échapper à la misère et aux difficultés de la période de l'après-guerre, ils sont largement diffusés malgré la censure et la condamnation du cosmopolitisme. Contrairement à ces films projetés sur l'ensemble du territoire soviétique, les pratiques d'écoute de la radio étrangère sont plus nombreuses dans les pays Baltes que dans d'autres républiques de l'URSS et cela pour une raison technique : le taux d'équipement des ménages en postes de radio y est le meilleur. Le brouillage commence en Estonie en 1949, mais son efficacité laisse à désirer. En même temps, l'interdiction d'écouter les stations étrangères ne fut jamais officiellement décrétée. Cette politique hésitante à l'égard des médias occidentaux permet à l'auteur de minimiser l'isolement de l'URSS en matière d'accès à l'information et par là de douter de l'étanchéité du rideau de fer.

L'idée de la circulation de l'information et des emprunts médiatiques d'un régime à l'autre est aussi au cœur de la contribution de Chloë Stephenson qui étudie l'intérêt suscité par le cinéma soviétique en Italie fasciste. L'exemple soviétique est perçu comme un art exaltant un nouvel ordre social, comme une industrie bien organisée produisant des œuvres d'art efficaces à des fins de propagande. Ainsi, c'est plutôt le modèle idéal qui est importé et non pas les pratiques réelles truffées de dysfonctionnements organisationnels, de problèmes de production des films faute de matériel et de l'accommodement de l'offre à la demande du public qui préfère des films de divertissement.

6 La question de la vocation des médias sous les régimes autoritaires est abordée dans les articles d'Inge Marszolek, de Pavlína Binková et d'Indrek Treufeldt. La nécessité de divertir dispute souvent le premier rôle à l'impératif de propagande. Comme le montre Inge Marszolek, la question juive est ainsi très peu présente dans les émissions radiophoniques de l'Allemagne nazie (sauf lors de la transmission des discours de Goebbels), car la radio pendant la période nazie est devenue un média divertissant plus qu'un média éducatif. Faire de la propagande antisémite à la radio pouvait, selon l'auteur, troubler la routine de la vie quotidienne. Pavlína Binková montre qu'en Tchécoslovaquie, la télévision ne devient un instrument d'éducation culturelle et politique que durant les années 1960, quand le nombre de postes dans les ménages atteint deux millions. Les années 1950 n'ont pas laissé de traces d'une politique systématique à l'égard de la télévision. Dans l'article sur la naissance des actualités télévisuelles en Estonie soviétique, Indrek Treufeldt, affirme que l'éducation politique allait de pair avec le divertissement. Les deux auteurs s'accordent tout de même sur les fonctions sociales de ce nouveau média dans les pays socialistes: la télévision devait créer l'illusion d'une cohésion sociale parfaite, en montrant des foules, des délégations, en parlant de «nous» et en évitant les oppositions, les thèmes et les images conflictuels.

7 L'aperçu des transformations des médias sous l'impact de la soviétisation et de la Guerre froide offert dans ce livre serait sans doute encore plus riche si ces transformations pouvaient être mises plus systématiquement en relation avec des changements sociétaux. On aimerait notamment savoir comment les mutations des médias affectent les rapports sociaux, les pratiques culturelles, les modes de consommation et l'imaginaire social et politique. Les analyses de la réception des discours médiatiques concurrents (socialistes et occidentaux) auraient pu être prolongées par une réflexion sur la construction des subjectivités. Cependant, même si 
le livre laisse le lecteur un peu sur sa faim, il sera utile à tous ceux qui s'intéressent au fonctionnement des médias dans les démocraties populaires et plus généralement à la construction du bloc soviétique. 